\title{
Measuring environmental tobacco smoke exposure in infants and young children through urine cotinine and memory-based parental reports: empirical findings and discussion
}

Georg E Matt, Dennis R Wahlgren, Melbourne F Hovell, Joy M Zakarian, John T Bernert, Susie B Meltzer, James L Pirkle, Samuel Caudill

\begin{abstract}
Objective-This study examined the reliability and potential biases of two urine collection methods from which cotinine measures were obtained and the validity of memory-based parental reports of their children's exposure to environmental tobacco smoke (ETS).

Design-Structured interviews were conducted with mothers of infants and young children to obtain memory-based estimates of recent ETS exposure. Urine samples were collected through standard and cotton roll collection methods for cotinine analysis.
\end{abstract}

Setting-All interviews took place at an off-campus research facility. Urine samples were collected at the study office or the subjects' homes.

Participants-Mothers were recruited from San Diego county sites of the Women, Infants, and Children (WIC) Supplemental Food and Nutrition Program. Sample 1 (infants) consisted of eight boys and eight girls aged 1-44 months $($ mean $=12.6$ months). Sample 2 (children) included 10 boys and 10 girls aged 3-8 years (mean $=61.2$ months).

Main outcome measures-Urine cotinine and memory-based parent reports of ETS exposure from structured interviews.

Results-There was overall high reliability for urine cotinine measures and no effect of collection method on urine cotinine levels. Memory-based reports obtained from smoking mothers showed moderately strong and consistent linear relationships with urine cotinine measures of their infants and children $(r=0.50$ to $r=0.63$ ), but not for reports obtained from non-smoking mothers.

Conclusions-Memory-based parental reports of short-term ETS exposure can play an important role in quantifying ETS exposure in infants and children.

(Tobacco Control 1999;8:282-289)

Keywords: environmental tobacco smoke; parental smoking; passive smoking

\section{Introduction}

Environmental tobacco smoke (ETS) refers to the diluted sidestream and exhaled smoke released into the atmosphere when cigarettes are smoked. Over the past decade, several reports have concluded that ETS exposure is linked to disease and death. ${ }^{1-3}$ ETS exposure in infancy and childhood is particularly harmful because it alters lung function and structure and creates changes known to predispose children to long-term pulmonary risks. ${ }^{2}$

Currently, there is no means by which the harmful components of ETS can be directly measured in the organs of interest-for example, the lungs. ${ }^{4}$ Therefore, researchers have developed indirect measures of ETS exposure, generally categorised as self-reports, biological markers, and environmental air monitors. These measures differ considerably in terms of reliability, validity, potential biases, cost, and ease of administration.

Because infants cannot provide reports of their ETS exposure, researchers have relied on memory-based proxy reports - by a parent, for example - of the presence or absence of a smoker living in the home, or parental, or other residents' smoking rates. ${ }^{5-7}$ Although parental reports of exposure magnitude of their older children (over six years old, for example) has demonstrated acceptable reliability and validity, ${ }^{89}$ the same has not yet been documented for young children and infants. In general, proxy reports are subject to the same sources of error and bias associated with self-reports and discussed in the literature. ${ }^{1}{ }^{10-12}$ Memory-based proxy reports, for instance, may be questioned because of the social desirability not to expose an infant to ETS, and because of limitations in the reporter's ability to estimate accurately the duration, proximity, or frequency of exposure, and to accurately report potentially important physical characteristics of the exposure setting, such as air ventilation or air conditioning. Nevertheless, memory-based proxy reports of ETS exposure in infants and young children may be the only available and affordable source of information about ETS exposure for epidemiological research and clinical practice.

Cotinine, the major proximate metabolite of nicotine, is the most widely used biological marker of ETS exposure and can be detected in saliva, blood, urine, semen, and hair. ${ }^{10} 1314$ Even though biological markers such as cotinine are unaffected by many of the sources of potential bias and error found in memory-based reports of ETS exposure, biomarkers are not without shortcomings.
Revision

Accepted 15 April 1999 
Although the presence of cotinine indicates that a person has been exposed to nicotine, the amount of cotinine may not be directly related to the amount of the disease-causing constituents, such as benzo(a)pyrene, to which a person was exposed. ${ }^{15}$ The concentration of cotinine in non-smokers is affected not only by ETS exposure but also by individual differences in the uptake, distribution, metabolism, and excretion of nicotine. In breastfeeding infants, cotinine concentration is also influenced by the mothers' frequency of breastfeeding, smoking behaviour, ETS exposure, and use of nicotine replacement therapies. ${ }^{16-18}$ In addition, the half life of cotinine renders these measures capable of estimating ETS exposure over a relatively short period of time (two to three days from fluids, possibly a few months from hair), whereas many health effects may result from long-term exposure $^{1}$ (months to years).

When measuring ETS exposure in the field through biological markers, intrusiveness and convenience are additional important considerations, and are further complicated when measuring exposure among infants. In general, measures of biological markers are more likely to be subject to sample contamination when obtained in the field rather than the lab. This is particularly a concern for detecting the presence of and measuring changes in low-level ETS exposure in infants. Measuring cotinine and nicotine concentration in hair samples may not be feasible in many infants with minimal new hair growth. Moreover, because nicotine and cotinine in hair samples provide a measure of longer term ETS exposure $^{19} 20$ (approximately one month of exposure per centimetre of hair) it is not suited for measuring very recent exposure or short-term change (three to four days).

Drawing blood in infants is frequently avoided because of difficulties in collecting sufficient amounts and because of the distress it causes infants and their parents. Saliva is a viable source of cotinine. It is relatively easy to collect in older children and adults, but more difficult to obtain from infants as the subject's cooperation is required. Salivary cotinine levels are similar to those in serum, so relatively large volumes (at least $1 \mathrm{ml}$ for a single analysis) are required for assessing low-level ETS exposure. Saliva is more subject to contamination than either serum or urine samples. Thus although saliva and serum are feasible alternatives, urine is often preferred for infant cotinine assays because it provides a more convenient and less intrusive means of collection. Because cotinine concentrations in various biological fluids are highly correlated $(r>0.80),{ }^{21} 22$ the concentration in urine provides a representative measure of exposure found in other biological fluids. ${ }^{10}$

For clinical and epidemiological research purposes the search for acceptable measures of ETS exposure has yet to be completed. Reliable and valid measures with at least interval-level scaling are needed to allow progress in determining minimum exposure levels for disease risk estimation, and to allow for more accurate assessment of change in ETS reduction trials. Development of field measures which are reliable, valid, economical, and practical for large-scale use remains to be accomplished.

This study contributes to the development of ETS exposure measures in infants and young children in the field by (a) examining the reliability of urine cotinine measures, (b) studying the potential effect of two urine collection methods on cotinine measures, and (c) exploring the relationship of urine cotinine measures with memory-based parental reports of ETS exposure in two samples of infants and children.

\section{Methods}

SUBJECTS

Sample 1: infants

Subjects were eight boys and eight girls aged 1-44 months (mean $=12.6$ months). Seven were Asian-American, five were non-Hispanic Anglo, one was Hispanic, one was African-American, and two children were of unknown ethnicity. Subjects were recruited from a WIC (Women, Infants, and Children Supplemental Food and Nutrition Program) site in San Diego county. Clients completed screening forms during regular WIC visits. Mothers with children from $0-4$ years were contacted by telephone and asked to participate. Breastfeeding mothers were excluded. Five mothers reported no ETS exposure over the past week, and eight mothers reported that they were non-smokers.

\section{Sample 2: children}

Subjects were 10 boys and 10 girls aged 3-8 years (mean $=61.2$ months). Nineteen children were of Hispanic ethnicity. The remaining child was non-Hispanic Anglo. Five subjects were recruited from a WIC site in San Diego county. These subjects' mothers provided referrals for 14 subjects, and one subject was recruited from a community clinic. All mothers reported that their children were exposed to ETS during the past week, and 13 mothers were non-smokers.

\section{URINE COLLECTION}

\section{Sample 1}

For infants wearing diapers (nappies) $(\mathrm{n}=11)$, urine samples were collected using a sterile paediatric urine collection bag (Pediabag 14-5501, Kendall Medical Products, Mansfield, Massachusetts). The bag was taped around the genital area, and the child's regular diaper was worn over the bag. For toilet-trained children ( $n=5)$, urine samples were collected using standard urine collection cups. To obtain a minimum of $30 \mathrm{ml}$ of urine, up to four voids were combined if necessary (seven of 16 infants). Although multiple voids were usually collected within three hours, voids collected up to three days apart had to be combined because of scheduling conflicts for three children. Samples were obtained at the study office $(n=10)$ or at subjects' homes $(n=6)$. Samples were frozen immediately when collected at the study office at $-20^{\circ} \mathrm{C}$, or immediately after returning to the office from 
home visits. In the instances where multiple voids were collected over a day apart, the previously collected sample was thawed to room temperature and combined with the new sample and then re-frozen.

Study personnel used a pipette to transfer urine collected from the Pediabag into two standard urine collection cups. Under the standard urine collection method, a sterile syringe was used to withdraw urine from the first cup and to express it into two sterile $5 \mathrm{ml}$ plastic vials, yielding two identical urine replications.

Cotton rolls placed in the diaper are the preferred means of obtaining urine samples in infants without the inconvenience and high failure rate-particularly in infant girls - of a pediatric collection bag. Therefore, it was important to test for potential sources of contamination caused by the use of cotton rolls. Samples of cotton rolls were pre-screened to confirm the absence of any detectable cotinine contamination. Under the cotton roll collection method, study personnel soaked a clean cotton dental roll in the urine from the second collection cup. Next the cotton roll was placed in a sterile 20 gauge syringe and expressed into two sterile $5 \mathrm{ml}$ plastic vials. This was repeated with a second cotton roll and a new syringe, yielding two replication samples.

\section{Sample 2}

The older children of sample 2 were asked to urinate into standard collection cups, yielding a minimum of $30 \mathrm{ml}$ of urine in a single void for all 20 children. Samples were obtained at the subjects' homes $(\mathrm{n}=19)$ or at the community clinic $(n=1)$. A sterile pipette was used to transfer equal amounts of urine from collection cups to four $10 \mathrm{ml}$ plastic test tubes, yielding four urine replications.

In summary, for infants in sample 1, cotinine values were reported for two urine bag samples and two cotton roll samples. For children in sample 2, cotinine values were reported for four collection cup samples.

\section{COTININE ANALYSES}

Samples were transported in an ice chest to the study office, where they were immediately frozen in a standard freezer $\left(-20^{\circ} \mathrm{C}\right)$. Each vial and test tube was labelled with a different randomly assigned identification number for laboratory use to mask samples collected from the same subjects. Batched samples were packed in dry ice and shipped via overnight delivery to the Air Toxicants Branch at the Centers for Disease Control and Prevention (CDC), Atlanta, Georgia, USA, for analysis. All samples were assayed by a modification of a high-performance liquid chromatography and atmospheric pressure chemical ionisation tandem mass spectrometry method (HPLC API MS/MS) that had been previously developed for the analysis of cotinine in serum. ${ }^{23}$

All samples were analysed twice in separate runs, and each run included duplicate water blank and urine pool control samples. All runs remained in control throughout this study, and all sample duplicates demonstrated good agreement. The target value for the urine control pool was $12.9 \mathrm{ng} / \mathrm{ml}$, and the observed result from all runs in this series was $12.97 \mathrm{ng} /$ $\mathrm{ml}$ (SD $0.663 \mathrm{ng} / \mathrm{ml}$ ), relative standard deviation $(\mathrm{RSD})=5.1 \%, \mathrm{n}=18$. The mean concentration from the duplicate assays was reported in each case for the unknown samples and all results were above the detection limit for this assay (approximately $0.05 \mathrm{ng} / \mathrm{ml}$ ).

MEMORY-BASED MEASURES OF TOBACCO EXPOSURE

The degree of exposure was operationalised as the number of cigarettes smoked in the same room as the child. Smoking mothers reported the child's exposure to ETS from herself and from all others in the home or elsewhere over the previous 48 hours and the previous week. Non-smoking mothers reported the child's exposure from the other parent and from all others in the home or elsewhere over the previous 48 hours and the previous week.

Mothers were told that their children's urine samples would be analysed to determine the amount of tobacco smoke to which their child had been exposed, thereby possibly enhancing the truthfulness and accuracy of memorybased measures. ${ }^{24}{ }^{25}$ The following memorybased measures of smoking and exposure were collected from mothers.

- 48-Hour total exposure: total number of cigarettes to which the child was reportedly exposed over the previous 48 hours

- One-week total exposure: total number of cigarettes to which the child was exposed over the previous week.

- 48-Hour parent exposure: number of cigarettes smoked by the "parent" to which the child was exposed over the previous 48 hours. If the mother smoked, this was a selfreport; otherwise, the mother estimated the exposure caused by the other parent.

- One-week parent exposure: number of cigarettes smoked by the "parent" to which the baby or child was exposed over the previous week. If the mother smoked, this was also a self-report; otherwise, the mother estimated the exposure caused by the other parent.

STATISTICAL ANALYSES

All data were checked for data-entry and copying errors before analysis with SPSS version 6.1 and SAS version 6.12. Outlying cotinine values were double-checked with the CDC laboratory to rule out clerical errors. For all statistical analyses, cotinine values were subjected to a $\log _{10}$ transformation to control for increased variability among higher cotinine values. Unless noted otherwise, cotinine values reported in the results section have been untransformed by exponentiation. Analyses of variance were conducted to assess potential effects of collection method on cotinine measures. Pearson product-moment correlations were computed to assess correspondence between parental reports of ETS exposure and urine cotinine measures. 


\section{Results}

RELIABILITY OF URINE COTININE MEASURE

Sample 1: cotton and standard collection methods in infants

To examine the reliability of the cotinine measures obtained from the cotton and standard collection methods, intra-class correlations ${ }^{26}$ were computed for the cotinine measures obtained from standard and cotton collection methods. The reliabilities are $\mathrm{Rel}=0.9968$ and Rel $=0.9845$, respectively. The corresponding standard errors of measurement (SEM $=\mathrm{s}_{\mathrm{x}}$ $\left.(1-R e l)^{1 / 2}\right)$ in $\log _{10}$ units are $\mathrm{SEM}_{\text {standard }}=$ $0.04434 \mathrm{ng} / \mathrm{ml}$ and $\mathrm{SEM}_{\text {cotton }}=0.09543 \mathrm{ng} / \mathrm{ml}$ for the standard and cotton roll collection methods, respectively. This translates into $95 \%$ confidence intervals (CIs) of 4.63 to 6.91 and 3.68 to 8.71 for individual cotinine measures at $5.66 \mathrm{ng} / \mathrm{ml}$ (mean in sample 1) using the standard and cotton methods, respectively. For an individual cotinine measure at $20 \mathrm{ng} / \mathrm{ml}$ (high exposure level ), 95\% CI $=16.37$ to 24.43 for the standard collection method and $95 \% \mathrm{CI}=13.00$ to 30.77 for the cotton collection methods. That is, the actual urine cotinine levels are expected to fall within these intervals in 95 out of 100 measures.

Sample 2: standard collection method in young children

The intra-class correlation is 0.9997 in sample 2 , yielding $\mathrm{SEM}=0.004928 \mathrm{ng} / \mathrm{ml}$. For individual cotinine measures of $2.06 \mathrm{ng} / \mathrm{ml}$ (average in sample 2), 95\% CI $=2.01$ to 2.11 . For an individual cotinine measure at $20 \mathrm{ng} / \mathrm{ml}$ (high exposure level), 95\% CI $=19.56$ to 20.45 .

EFFECTS OF COTTON ROLL $V$ STANDARD URINE COLLECTION METHODS

To examine whether the cotton roll method yields cotinine levels that differ systematically from those obtained from the more reliable standard collection method, a 2 (urine collection method) $\times 2$ (replication) repeated measures analysis of variance was conducted in sample 1 . The table shows sample means and standard deviations. This analysis revealed no statistically significant method main effect $(F(1,15)=0.76 ; \mathrm{p}=0.40)$, suggesting that using cotton rolls to collect urine does not systematically affect level of urine cotinine measured.

It should be noted that the relatively small sample size underlying this analysis $(n=16)$

Descriptive statistics for urine cotinine replications (in $\mathrm{ng} / \mathrm{ml}$ ) for sample 1 (16 infants), sample 2 (20 children), and samples 1 and 2 combined

\begin{tabular}{lllll}
\hline Variable & Mean & $S D$ & Minimum & Maximum \\
\hline Sample 1 & & & & \\
$\quad$ Cotton roll 1 & 5.25 & 6.03 & 0.1700 & 52.5 \\
$\quad$ Cotton roll 2 & 5.50 & 6.31 & 0.0850 & 44.3 \\
$\quad$ Standard 1 & 5.89 & 5.75 & 0.1170 & 51.3 \\
$\quad$ Standard 2 & 6.03 & 5.62 & 0.0740 & 53.5 \\
Sample 2 & & & & \\
$\quad$ Replication 1 & 2.48 & 3.36 & 0.1760 & 65.8 \\
$\quad$ Replication 2 & 2.47 & 3.32 & 0.1410 & 65.3 \\
$\quad$ Replication 2 & 2.43 & 3.38 & 0.1750 & 67.8 \\
$\quad$ Replication 3 & 2.45 & 3.34 & 0.1560 & 66.0 \\
\multicolumn{2}{l}{ Samples 1 and 2 combined } \\
$\quad$ Pooled replications & 3.56 & 4.59 & 0.1695 & 66.2 \\
\hline
\end{tabular}

may have prevented us from detecting small but existing differences caused by the collection method. The observed means (see table) suggest that such effects-if they existed-are likely to be very small $(<0.12$ standard deviation units or $<0.78 \mathrm{ng} / \mathrm{ml}$ ). Whether an effect of this size has clinical relevance depends on its relationship with health outcomes and cannot be answered by this study.

RELATIONSHIP BETWEEN URINE COTININE AND MEMORY-BASED REPORTS OF ETS

To examine whether parents can provide valid reports of the amount of ETS to which their infants and children were exposed, we correlated the parents' memory-based reports with their children's urine cotinine levels. Because the data collected in sample 1 do not suggest a bias in cotinine levels introduced by the collection method, we averaged the cotinine measures from the four replications, yielding a mean urine cotinine level per person. Similarly, the cotinine measures from the four replications of the standard collection method in sample 2 were averaged to obtain a mean urine cotinine level per person. To increase statistical power, we then combined the two samples for a total sample size of $n=36$. Pooling data from the two samples can be justified on the following grounds: the absence of a method effect on cotinine levels in sample 1 yields four equivalent cotinine measures per subject and thus equivalent data structures in samples 1 and 2 . Urine cotinine levels are not significantly $(p>0.30)$ associated linearly or quadratically with age in sample 1 or in sample 2 .

\section{8-Hour total exposure}

Figure 1 shows a scatterplot of urine cotinine and the memory-based estimates of the total number of cigarettes to which the child was exposed over the previous 48 hours. Parental reported total exposure over the previous 48 hours ranged from none to 92 cigarettes, with a median exposure level of 6.5 cigarettes (mean $=18.4$ ). The linear relationship between urine cotinine and memory-based reports was $r=$ $0.2760\left(r^{2}=0.0762 ; \mathrm{p}=0.103\right)$ with a cluster of potential "false-positive cases" (low urine cotinine and high memory-based exposure report) as well as some potential "false-

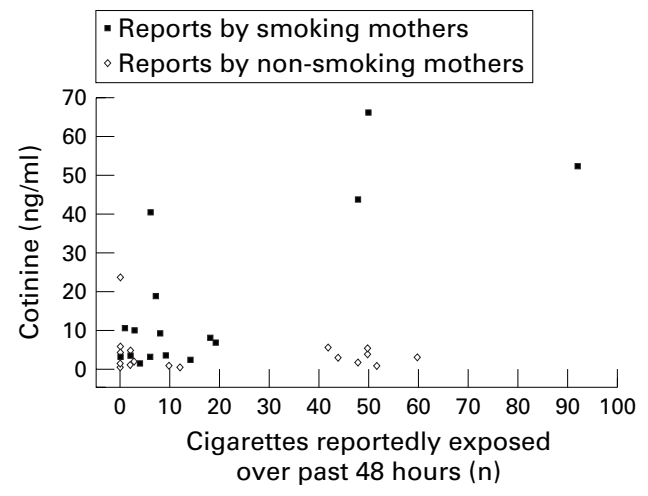

Figure 1 Scatterplot of mean urine cotinine ( $\mathrm{ng} / \mathrm{ml}$ ) and memory-based reports of the total number of cigarettes to which the child was exposed over the previous 48 hours. 
negative" cases (high urine cotinine and low memory-based exposure report).

Closer examination revealed that all seven potential false-positive cases involved nonsmoking mothers reporting exposure caused by others (indicated by the open circle in figure 1). That is, non-smoking mothers tended to report higher levels of exposure than one would expect based on the levels of cotinine observed in the infants' urine. Alternatively, urine cotinine levels from children of non-smoking mothers are lower than one would expect based on the mothers' reported exposure levels (see figure 1). When reports by non-smoking mothers were excluded from the analyses, the proportion of shared variance between urine cotinine levels and memory-based exposure levels increased substantially to $r^{2}=0.4022(r=0.6342 ; \mathrm{p}=$ 0.008 ). Total exposure over the previous 48 hours for this subsample ranged from none to 92 with a median exposure level of 7.5 cigarettes (mean 17.9).

To examine how well urine cotinine levels and memory-based reports can be predicted from each other (excluding non-smoking parents), the standard errors of estimates (SEE) were obtained $\left(\mathrm{SEE}=\mathrm{s}_{\mathrm{Y}}\left(1-r_{\mathrm{XY}}^{2}\right)^{1 / 2}\right)$. They were $\mathrm{SEE}=0.4068$ for predicting cotinine from parental reported ETS exposure and SEE $=19.24$ for predicting the parental reported ETS exposure based on urine cotinine. For a predicted cotinine level of an individual subject at an average exposure level $(8.85 \mathrm{ng} / \mathrm{ml})$, the standard error of estimate yielded a $95 \%$ CI of 1.4 to 55.5. That is, if memory-based reports of total exposure over the previous 48 hours were used to predict urine cotinine at 8.85 , in $95 \%$ of these predictions the observed urine cotinine level would fall between $1.4 \mathrm{ng} / \mathrm{ml}$ and $55.5 \mathrm{ng} /$ $\mathrm{ml}$. Correspondingly, predicting number of cigarettes to which the child was reportedly exposed based on urine cotinine yielded a 95\% CI of 0 to 55.6.

We also explored the relationship between creatinine-adjusted cotinine measures and memory-based parental reports. However, creatinine-adjusted cotinine showed systematically lower correlations with parental reports than the unadjusted cotinine measures-for example, $r=0.40 v r=0.49$ for ETS exposure over the previous 48 hours-even though the correlation between creatinine-adjusted and unadjusted cotinine measures was $r=0.84$.

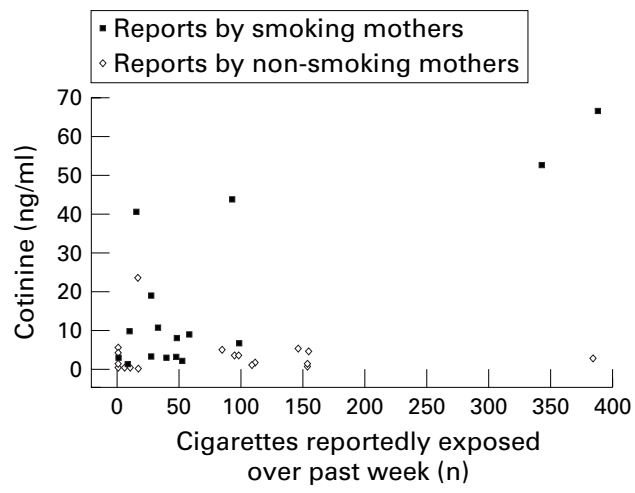

Figure 2 Scatterplot of mean urine cotinine $(\mathrm{ng} / \mathrm{ml})$ and total exposure to cigarettes over the previous week.
Because the unadjusted cotinine measures appear to be more closely related to parents' memory-based reports of ETS exposure, we are reporting validity analyses based on the unadjusted cotinine measures.

\section{Total exposure over the previous week}

Figure 2 shows a scatterplot of urine cotinine and the memory-based estimates of the total number of cigarettes to which the child was reportedly exposed during the previous week, excluding reports from non-smoking parents. Total exposure over the previous week ranged from none to 388 with a median exposure level of 43 cigarettes (mean 80.2). The linear relationship between urine cotinine and memory-based reports was $r=0.6164\left(r^{2}=\right.$ $0.3799 ; p=0.011)$.

\section{Exposure to parents' cigarettes over the previous 48 hours}

Exposure over the previous 48 hours to the cigarettes smoked by parents ranged from none to 20 cigarettes with a median exposure level of 3.5 and a mean of 5.0 cigarettes. The linear relationship between urine cotinine and memory-based reports was $r=0.5660\left(r^{2}=\right.$ $0.3204 ; \mathrm{p}=0.022)$.

Exposure to parents' cigarettes over the previous week

Exposure to parents' cigarettes only, over the previous week, ranged from none to 105 with a median exposure level of 17.5 cigarettes (mean $=23.9$ ). The linear relationship between urine cotinine and memory-based reports was $r=$ $0.5002\left(r^{2}=0.2504, \mathrm{p}=0.048\right)$.

\section{Discussion}

\section{OVERALL RELIABILITY}

Urine cotinine analyses using urine samples collected under field conditions and analysed using HPLC API-MS/MS were highly reliable. Overall reliability estimates indicate that less than $2 \%$ of the observed variance in cotinine was due to measurement error. The standard error of measurement was lowest in sample 2 (SEM = $0.004928 \mathrm{ng} / \mathrm{ml}$ in $\log _{10}$ units) and highest for the cotton roll method used in sample 1 (SEM = 0.09543). Consequently, the $95 \%$ confidence intervals for specific contine measures based on the cotton roll method are larger than those based on the standard method. It appears that in the handling of cotton rolls (soaking, extracting), sources of measurement error were introduced that were not present in the standard collection method. A review of collection and testing methods did not lead to hypotheses that might account for these discrepant cases. Further investigation of individual measures revealed four cases in sample 1, in which the within-subject standard deviation across the four replications exceeded $3 \mathrm{ng} / \mathrm{ml}$, whereas it was less than $1.5 \mathrm{ng} / \mathrm{ml}$ for all the others. Thus, it appears that only a subset of cases was influenced by substantial measurement errors, calling for improvements in standardisation of field procedures for collecting, handling, and extracting cotton rolls. 
Despite the somewhat lower reliability of cotinine measures collected with the cotton roll procedure, the achieved reliabilities yield a level of measurement precision that should serve well in most field research of low-level ETS exposure in children. Based on the cotton roll method used in this study, a typical exposure level of an infant at $5.66 \mathrm{ng} / \mathrm{ml}$ can be reliably distinguished (with 95\% confidence) from $3 \mathrm{ng} / \mathrm{ml}$ and $9 \mathrm{ng} / \mathrm{ml}$, and an exposure level of $20 \mathrm{ng} / \mathrm{ml}$ can be reliably distinguished from 13 and $31 \mathrm{ng} / \mathrm{ml}$.

Whether this level of precision is sufficient depends on the intended use of the measures, the relationship between exposure to cigarettes and urine cotinine, and the dose-response relationship. For instance, if this measure were to be used to screen for infants and children with high ETS exposure-for example, $>40.0 \mathrm{ng} /$ $\mathrm{ml}$ - the observed margin of error will be small enough to aid in this decision.

If the task is to examine dose-response relationships across a wide range of ETS exposure (as was the case in the present study with reported exposures ranging from 1 to 388 cigarettes per week and urine cotinine ranging from 0.09 to $67.8 \mathrm{ng} / \mathrm{ml}$ ), the observed precision will also be adequate. However, if the purpose is to examine dose-response relationships carefully within a narrow range of exposure, such as low exposure-5.0 to $10.0 \mathrm{ng} / \mathrm{ml}$, the observed precision may be inadequate. For such a task, the more reliable standard collection method will be the method of choice.

COLLECTION METHOD

Urine cotinine measures do not appear to be systematically influenced by using cotton rolls when collecting urine from infants compared with standard urine collection bags. That is, we found no evidence suggesting a bias in urine cotinine levels caused by the collection methods. Even though this finding has to be interpreted in light of low statistical power, the observed difference $(0.78 \mathrm{ng} / \mathrm{ml})$ between the highest and the lowest cotinine means suggest no clinically important difference, especially given the overall reliability of the cotton roll and standard urine cotinine measures.

VALIDITY OF MEMORY-BASED PARENT REPORTS Memory-based reports of ETS exposure by smoking mothers showed moderately strong linear relationships with urine cotinine levels of their small children. However, this was not the case for reports made by non-smoking mothers who tended to report higher exposure levels than one would expect based on observed cotinine levels. After excluding reports by non-smoking mothers, memory-based reports of total ETS exposure over the previous 48 hours accounted for $40.2 \%$ of the variance in urine cotinine levels of their children, and $38.0 \%$ for total exposure over the previous week. The corresponding figures for exposure caused by parents alone are $32.0 \%$ (previous week) and $25.0 \%$ (previous 48 hours).

We suspect that the differential validity of memory-based exposure reports may reflect the operation of several phenomena. First, in homes of smoking mothers, children are likely to be exposed to ETS at closer proximity than in homes where people other than the mothers smoke. Because of the closer proximity, higher urine cotinine levels are observed in children whose mothers contributed to the exposure. That is, mothers may actually give accurate reports of the number of cigarettes smoked in the presence of the child. However, the number of cigarettes smoked in the presence of the child is a more valid indicator of actual exposure (clinically significant inhalation of ETS) in smoking mothers than it is in non-smoking mothers. Second, exposure reports by non-smoking mothers may be inferior because smoking episodes may not be encoded very reliably and because a few but salient exposure episodes may be given too much weight when estimating overall exposure. Third, some non-smoking mothers may exaggerate reports of exposure as a means of influencing a smoker to quit smoking or to reduce the child's exposure.

Because exposure reports by non-smoking mothers showed no correlation with urine cotinine, their reports attenuated the relationship between urine cotinine and memory-based reports of ETS exposure when analysed together with reports from smoking parents. Thus, it becomes critical to take into account the source for memory-based ETS reports when using such reports for screening purposes in clinical practice, as outcome measures in intervention trials, or in epidemiological field research.

LIMITATIONS AND IMPLICATIONS

First, although our findings provide encouraging support for the reliability of field methods for urine cotinine measures and the validity of memory-based parent reports of the magnitude of ETS exposure, the size and nature of this sample limits the generalisability of our findings to other populations, settings, and interview modalities. Particularly, conclusions about non-smoking parents' reports were based on a very small number of participants and require further examination. Replications and further investigations of the proposed methods in larger probability samples are clearly indicated.

Second, the strength of the relationship observed in our sample clearly benefited from the observed range of ETS exposure. Excluding reports by non-smoking mothers, approximately $25 \%$ of infants and children were reportedly exposed to 14 or more cigarettes and $10 \%$ to more than 40 cigarettes over a 48-hour period. In samples with more restricted ranges of exposure, the relationship between number of cigarettes exposed and urine cotinine will be attenuated, ${ }^{27}$ and a much larger sample may be required to achieve sufficient statistical power to detect such small relationship.

Third, while we observed moderately strong positive relationships between reported ETS exposure and urine cotinine, the strength of these relationships does not permit accurate 
predictions of the urine cotinine levels or the number of cigarettes exposed for an individual subject. That is, while there is general correspondence between urine cotinine and parental reports of exposure $(30-40 \%$ shared variance), the two measures are far from providing identical information about ETS exposure at the level of the individual person $(60-70 \%$ of the variance is not shared). To better account for the residual variance, future research has to take into account interindividual difference variables affecting the uptake, metabolism, and secretion of nicotine as well as limitations in parental reports of ETS exposure-for example, incentives to under or overestimate exposure - and a host of variables characterising in more detail the nature of exposure, such as proximity to exposure source, duration of exposure, ventilation rates, and type of cigarettes.

Fourth, memory-based recall in this study covered the previous 48 hours and the previous week. The 48-hour time frame is particularly appropriate to validate against urine cotinine measures because of the metabolite's half-life ${ }^{28}$ (40-60 hours). Although our findings provide empirical support for the validity of short-term exposure reported by parents, this study does not speak to the validity of longer-term exposure.

Fifth, though non-smoking mothers tended to report much higher exposure levels than the urine cotinine of their children suggested, this may not be a fatal flaw in all studies. For some epidemiological studies or clinical trials, this error, if stable, might not preclude the use of memory-based measures as indicators of change or of group differences. One option for dealing with this issue involves augmenting reports from non-smoking parents through collaborating evidence from the smoking parent. Another option relies on statistically modelling the smoking status of the reporting parent through, for instance, the inclusion of covariates such as smoking status of the responding parent. Another strategy would involve asking the non-smoking respondent to report only about directly observed ETS exposure, omitting speculations about ETS exposure while the respondent was absent. These possibilities should be considered in future studies of ETS exposure, especially for families in which the father is the smoker and the mother is the non-smoking caretaker (carer) likely to cooperate in epidemiological studies.

For epidemiological studies to use urine assays in infants, practical means of obtaining urine samples must be available and simple enough for parents or non-professional staff to use. They should also be equally applicable to infant boys and girls. One simple means of obtaining urine samples from infants, without requiring special efforts from parents or distressing the child, involves the placement of cotton rolls in the diaper. Research in other areas has suggested that urine assay results may vary according to collection methods when applied to assessments for infections, antigens, or exposure to illicit drugs. ${ }^{29-32}$ This study sug- gests that urine expressed from cotton rolls yielded essentially equivalent levels of cotinine when compared with urine collected directly from a collection bag. The present results suggest that cotton rolls are a practical means of obtaining urine samples for cotinine analyses without introducing biased observations.

Cotinine is often regarded as the gold standard measure of tobacco smoke exposure. ${ }^{10}$ However, it has been argued that cotinine is not an ideal gold standard for validating questionnaires. ${ }^{33}$ Cotinine data do not account for individual differences in physiology, such as metabolic rate, and do not inform when exposures occurred and at what magnitude on each occasion. For disease risk estimation and behaviour change purposes, it may be critical to determine the patterns in which exposure occurs. ${ }^{34-36}$ Biological measures alone will not provide this information; thus, ultimately, multiple convergent measures including combinations of biological, environmental/observational, and memory-based techniques may be required for ETS studies. ${ }^{37} 38$

All parents in this study were aware of the urine testing and were told that this test would determine the amount of tobacco smoke to which their infant was exposed. From the literature on the "bogus pipeline" phenomenon, it is known that when subjects are informed - or led to believe-that a self-report (such as number of cigarettes smoked) will be validated against presumably more objective evidence, the validity of the report tends to increase compared with the absence of a presumed objective confirmation. ${ }^{2425}$ Because all parental reported measures were collected in the context of accompanying urine testing, the observed validity of the memory-based ETS reports cannot be presumed in the absence of a real or "bogus" objective measure that parents believe will be used to confirm their reports of ETS exposure. ${ }^{939}$ Further research on the "bogus pipeline" effect on the validity of reported ETS exposure is needed to determine its general utility in clinical and epidemiological studies. Potentially, subjects could be routinely informed of the possibility that their responses may be verified through objective means, and such techniques might be occasionally administered even if assays will not be performed. The combination of memory-based ETS reports with intermittent confirmation by biological assays could set the stage for reliable and valid memory-based measures of ETS exposure that could be used in large-scale studies of tobacco control and for additional explorations of the effects of ETS exposure on health outcomes.

This research was supported in part by grants from the Smoke Free Families Program, Robert Wood Johnson Foundation, grant 027946, the Maternal and Child Health Bureau, United States Health Resources and Services Administration, grant 1 MCJ-060634-01-0, and the National Heart, Lung, and Blood Institu-06034-01-0, and the National Heart, Lung, and Blood Institute, National Institutes of Health, grant 1 R18 HL5283501A2, and by the United States Centers for Disease Control and Prevention. The authors would like to thank Alicia Vera and Susanna Concha-Garcia for their assistance with recruitment and data collection, and the reviewers and editor for their help-
ful suggestions.

1 California Environmental Protection Agency, Office of Environmental Health Hazard Assessment. Health effects of 
exposure to environmental tobacco smoke. Sacramento: California Environmental Protection Agency, September 1997.

2 US Environmental Protection Agency. Respiratory health effects of passive smoking: lung cancer and other disorders. Smoking and Tobacco Control Monograph No 4 Bethesda, Maryland: US Department of Health and Human Services, Public Health Service, National Institutes of Health, 1993. (NIH Publication No 93-3605.)

3 National Research Council Committee on Passive Smoking. Environmental tobacco smoke: measuring exposures and assessing health effects. Washington, DC: National Academy Press, 1986.

4 Nafstad P, Botten G, Hagen JA, et al. Comparison of three methods for estimating environmental tobacco smoke exposure among children aged between 12 and 36 months. Int $\mathcal{F}$ Epidemiol 1995;24:88-94.

5 Lyons RA. Passive smoking and hearing loss in infants. Ir Med f 1992;85:111-2.

6 Bisgaard H, Dalgaard P, Nyboe J. Risk factors for wheezing during infancy. A study of 5,953 infants. Acta Paediat Scand 1987;76:719-26.

7 Chen Y. Environmental tobacco smoke, low birth weight, and hospitalization for respiratory disease. Am $\mathcal{F}$ Respir Crit Care Med 1994;150:54-8.

8 Fried PA, Perkins SL, Watkinson B,et al. Association between creatinine-adjusted and unadjusted urine coti-
nine values in children and the mother's report of exposure nine values in children and the mother's report of exposure to environm

9 Emerson JA, Hovell MF, Meltzer SB, et al. The accuracy of environmental tobacco smoke exposure measures among asthmatic children. F Clin Epidemiol 1995;48:1251-9.

10 Benowitz NL. Cotinine as a biomarker of environmental tobacco smoke exposure. Epidemiol Rev 1996;18:188-204.

11 Leaderer BP. Assessing exposures to environmental tobacco smoke. Risk Anal 1990;10:19-26.

12 Repace JL, Jinot J, Bayard S, et al. Air nicotine and saliva cotinine as indicators of workplace passive smoking exposure and risk Risk Anal 1998;18:71-83.

13 Pacifici R, Altieri I, Gandini L, et al. Environmental tobacco smoke: nicotine and cotinine concentration in semen. Environ Res 1995;68:69-72.

14 Klein J, Chitayat D, Koren G. Hair analysis as a marker for fetal exposure to maternal smoking (letter). $N$ Engl f Med 1993;328:66-7.

15 Sanner T, Dybing E. [Health damages from passive smoking (see comments)]. Tidsskr Nor Laegeforen 1996;116:61720.

16 Stepans MB, Wilkerson N. Physiologic effects of maternal smoking on breast-feeding infants. $\mathcal{F}$ Am Acad Nurse Pract 1993;5:105-13.

17 Hardee GE, Stewart T, Capomacchia AC. Tobacco smoke xenobiotic compound appearance in mothers' milk after involuntary smoke exposures. I. Nicotine and cotinine. Toxicol Lett 1983;15:109-12.

18 Schulte-Hobein B, Schwartz-Bickenbach D, Abt S, et al. Cigarette smoke exposure and development of infants throughout the first year of life: influence of passive smoking and nursing on cotinine levels in breast milk and infant's urine. Acta Paediatr 1992;81:550-7.

19 Zahlsen K, Nilsen OG. Nicotine in hair of smokers and non-smokers: sampling procedure and gas chromatographic/mass spectrometric analysis. Pharmacol Toxicol 1994;75:143-9

20 Kintz P. Gas chromatographic analysis of nicotine and cotinine in hair. F Chromatogr 1992;580:347-53.
21 Jarvis M, Tunstall-Pedoe H, Feyerabend C, et al. Biochemical markers of smoke absorption and self reported cal markers of smoke absorption and self reported exposure to passive smo

22 Curvall M, Elwin CE, Kazemi-Vala E, et al. The pharmacokinetics of cotinine in plasma and saliva from non-smoking healthy volunteers. Eur $\mathcal{F}$ Clin Pharmacol 1990;38:281-7.

23 Bernert JT Jr, Turner WE, Pirkle JL, et al. Development and validation of sensitive method for determinatior cotinine in smokers and nonsmokers by liquid chromatography/atmospheric pressure ionization tandem mass spectrometry. Clin Chem 1997;43:2281-91.

24 Jones EE, Sigall $\mathrm{H}$. The bogus pipeline: a new paradigm for measuring affect and attitude. Psychol Bull 1971;76:34964.

25 Murray DM, O'Connell CM, Schmid LA, et al. The validity of smoking self-reports by adolescents: a reexamination of the bogus pipeline procedure. Addict Behav 1987;12:7-15.

26 Rosenthal R, Rosnow RL. Essentials of behavioral research: methods and data analysis. New York: McGraw-Hill, 1984.

27 Cohen J, Cohen P. Applied multiple regression correlation analysis for the behavioral sciences, 2 nd ed. Hillsdale, New analysis for the behavioral sciences, 2

28 Collier AM, Goldstein GM, Shrewsbury RP, et al. Urine cotinine elimination half-life in young children exposed to sidestream cigarette smoke. Indoor Air '90: health effects associated with indoor air contaminants. International conference on indoor air quality and climate, Toronto 1990. Ottawa, Ontario: Canada Mortgage and Housing Corporation, 1990:195-200

29 Cohen HA, Woloch B, Linder N, et al. Urine samples from disposable diapers: an accurate method for urine cultures. 7 Fam Pract 1997;44:290-2

30 Burke N. Alternative methods for newborn urine sample collection. Pediatr Nurs 1995;21:546-9. [Erratum in Pediatr Nurs 1996;22:172.]

31 Lombardero N, Casanova O, Behnke M, et al. Measurement of cocaine and metabolites in urine, meconium, and of cocaine and metabolites in urine, meconium, and Clin Lab Sci 1993;23:385-94.

32 Schlager TA, Hendley JO, Dudley SM, et al. Explanation for false-positive urine cultures obtained by bag technique. Arch Pediatr Adolesc Med 1995;149:170-3.

33 Idle JR. Titrating exposure to tobacco smoke using cotinine- a minefield of misunderstandings. 7 Clin Epidemiol 1990;43:313-7.

34 Emerson JA, Wahlgren DR, Hovell MF, et al. Parent smoking and asthmatic children's exposure patterns: a behavioral epidemiology study. Addict Behav 1994;19:677-89.

35 Robinson JP, Switzer P, Ott WR. Microenvironmental factors related to Californians' potential exposure to environmental tobacco smoke (ETS). Stanford, California: Department of Statistics, Sequoia Hall, Stanford University, 1994.

36 Robinson JP, Switzer P, Ott W. Daily exposure to environmental tobacco smoke: smokers vs nonsmokers in California. Am 7 Public Health 1996;86:1303-5.

37 Chan CC, Chen SC, Wang JD. Relationship between indoor nicotine concentrations, time-activity data, and urine cotinine-creatinine ratios in evaluating children's exposure to environmental tobacco smoke. Arch Environ Health 1995;50:230-4

38 Leaderer BP, Lioy PJ, Spengler JD. Assessing exposures to inhaled complex mixtures. Environ Health Perspect 1993; 101(suppl 4):167-77

39 Hovell MF, Meltzer SB, Zakarian JM, et al. Reduction of environmental tobacco smoke exposure among asthmatic children: a controlled trial.Chest 1994;106:440-6. [Erratum in Chest 1995; 107:1480.] 